\title{
Disulfide relays and phosphorylative cascades: partners in redox-mediated signaling pathways
}

\author{
G Filomeni $^{\star,}$, G Rotilio $^{1}$ and MR Ciriolo ${ }^{1}$ \\ ${ }^{1}$ Department of Biology, University of Rome 'Tor Vergata', Via della Ricerca \\ Scientifica, 00133 Rome, Italy \\ * Corresponding author: G Filomeni, Department of Biology, University of Rome \\ 'Tor Vergata', Via della Ricerca Scientifica, 1, 00133 Rome, Italy. \\ Tel: + 39-06-7259-4360; Fax: + 39-06-7259-4311; \\ E-mail: filomeni@bio.uniroma2.it
}

Received 26.5.05; accepted 13.7.05; published online 09.9.05 Edited by B Zhivotovsky

\begin{abstract}
Modifications of specific amino-acid residues of proteins are fundamental in order to modulate different signaling processes among which the cascade of phosphorylation represents the most effective example. Recently, also, the modification of the redox state of cysteine residues of certain proteins, which is a widespread mechanism in the regulation of protein function, has been proposed to be involved in signaling pathways. Growing evidence shows that some transcription factors could be modulated by both oxidation and phosphorylation. In particular, the pathways regulated by the mitogen activated protein (MAP) kinases represent well-established examples of the cross talk between redoxmediated signaling and phosphorylative cascades. This review will compare the two modes of signal transduction and propose an evolutionary model of a partnership of the two mechanisms in the eukaryotic cell, with redox-mediated signals being more specific and ancestral and phosphorylative signals being more diffuse but predominant in signal propagation.

Cell Death and Differentiation (2005) 12, 1555-1563.

doi:10.1038/sj.cdd.4401754; published online 9 September 2005
\end{abstract}

Keywords: glutathione; MAP kinases; signal transduction; phosphorylation; disulfides; redox

Abbreviations: ROS, reactive oxygen species; SOD, superoxide dismutase; GSH, reduced glutathione; GSSG, glutathione disulfide; GS-R, glutathione mixed disulfide with protein thiols; Trx, thioredoxin; NO, nitric oxide; GRX, glutaredoxin; RNS, reactive nitrogen species; Yap1, yeast activating protein 1; NES, nuclear export signal; AP-1, activating protein 1 ; NF- $\kappa$ B, nuclear factor- $k \mathrm{~B}$; HSF1, heat shock factor 1; Ref-1, Redox factor 1; STPK, serine/threonine protein kinases; MAP kinase, mitogenactivated protein kinase; TGF- $\beta$, tumor growth factor- $\beta$; PTK, protein tyrosine kinase; EGF-R, epidermal growth factor receptor; VEGF-R, vascular endothelial growth factor receptor; IGF-R, insulin-like growth factor receptor; PKC, protein kinase C; CDK, cyclin dependent kinase; JNK, c-Jun-N-terminal kinase; Bcl-2, B-cell lymphoma-2; MDM2, murine double minute 2; ASK1, apoptosis signal-regulating kinase 1; MKK, MAP kinase kinase; GST, glutathione-S-transferase; PTPase, protein tyrosine phosphatases

\section{Redox signaling}

The discovery in the late 1960s of the catalytic function of erythrocuprein as the dismutase of superoxide anion ${ }^{1}$ revolutionized the study of the biological role of $\mathrm{O}_{2}$ giving rise to the exponentially growing field of research on oxidative stress. In this perspective, oxygen is not only the ultimate electron sink of redox processes downstream consumption of carbon molecules and the source of energy coming from electron transport chain within the mitochondria, but, owing to its intermediate redox species (the free radicals $\mathrm{O}_{2} \bullet^{-}, \mathrm{OH}^{\bullet}$ plus $\mathrm{H}_{2} \mathrm{O}_{2}$ referred as ROS, reactive oxygen species), it represents a potential source of cellular damage. ${ }^{2}$ ROS are produced, even under physiological conditions, during the reduction of oxygen by the catalysis of enzymes (oxidases and oxygenases) as well as at the mitochondrial level by the Complex I and III of the electron transport chain. ${ }^{2}$ Their unconstrained effects give rise to oxidation and irreversible damage of unsaturated fatty acid of membranes, amino-acid residues of proteins, and nucleotide bases. ${ }^{2}$

The so-called antioxidant enzymes that remove the intermediates of oxygen reduction, in particular, superoxide dismutase (SOD), catalase and glutathione peroxidase act in damage control by preventing initiation and/or propagation of oxidizing radical chain reactions together with noncatalytic redox-active molecules. The latter ones can be divided into two different classes: (i) non-thiol compounds, essentially vitamins (e.g. ascorbate, tocopherols, $\beta$-carotene) and polyphenols, which are able to block the radical chain reaction by delocalizing the positive charge formed upon the interception of radicals; (ii) sulfur-containing compounds, such as glutathione (GSH) and thioredoxin (Trx), which act as donors of reducing equivalents either directly or in concert with specific enzymes.

It should be kept in mind that redox reactions occur not only during antioxidant responses to oxidative stimuli, but as the continuous fine adjustment of a network of redox couples linked to each other in response of electron movements within the cell, than the ultimate redox potential of the cell results from the contribution of the redox state of each couple. Glutathione (GSSG/GSH), nicotinamide adenine dinucleotide phosphate $\left(\mathrm{NADP}^{+} / \mathrm{NADPH}\right)$, and thioredoxin [TrxSS/ $\operatorname{Trx}(\mathrm{SH})_{2}$ ] couples are among the most important in maintaining an intracellular reducing environment. ${ }^{3}$ Actually, since the GSH concentration is 100 - to 10000 -fold higher than the reduced form of the other couples, the glutathione redox 
state usually determines the steady-state value of the intracellular redox potential. ${ }^{4} \mathrm{NADPH}$ and Trx redox couples play a concerted role to kinetically support a constant value of the GSH/GSSG ratio (Figure 1). The enzyme glutathione reductase (GSSG-Red) catalyzes the reduction of GSSG by using the NADPH-supplied reducing equivalents; on the other hand, Trx participates as electron donor in the reduction of mixed disulfide that may have formed between $\mathrm{GSH}$ and protein thiols (GS-R). ${ }^{4}$

We can distinguish two different modalities of oxidative alterations in the cell (Table 1). The first one derives from abrupt ROS overproduction: its prototype is the oxidative burst in response to bacterial infection during which phagocytes produce $\mathrm{O}_{2}{ }^{\bullet-}$ at high rates through the activation of a plasma membrane-associated NADPH oxidase. ${ }^{2}$ Another condition where ROS could be produced at a high level is in the case of xenobiotics- or drugs-mediated redox reactions. ${ }^{5}$ These compounds include quinones, bipyridils, antracyclins and nitro-imidazol molecules that, due to their chemical structure, easily undergo one-electron redox cycling with oxygen, giving rise to $\mathrm{O}_{2}{ }^{\bullet-}$ production. Usually, this explosive type of redox changes leads to irreversible cell damage and ultimately may give rise to necrotic cell death. The other type of oxidative alteration is due to physiological mild oxidative unbalances, which may be responsible for regulatory processes. In fact, stimuli of different origin, such as ligand/receptor binding, ${ }^{6}$ cytokines, ${ }^{7}$ UV $^{8}$ and osmotic shock, ${ }^{9}$ could result in localized, site-specific generation of ROS that escape the antioxidant defense by transferring their oxidizing equivalent to reactive cysteine residues of proteins, which are present as the thiolate form under physiological $\mathrm{pH}$. Depending on the surrounding environment sulfenic $(\mathrm{R}-\mathrm{SOH})$, or sulfinic $\left(\mathrm{R}-\mathrm{SO}_{2} \mathrm{H}\right)$, or sulfonic $\left(\mathrm{R}-\mathrm{SO}_{3} \mathrm{H}\right)$ acid derivatives are formed. ${ }^{10,11}$ The last two oxidative modifications irreversibly modify the structure and function of the protein involved, while sulfenic acid can be either reduced back to the thiolate form or transformed in a number of thiol adducts by reactions such as $S$-nitrosylation (addition to NO) or $S$-thiolation (disulfide bridge formation with other protein thiols or with $\mathrm{GSH}$ ). They represent stable and reversible derivatives of reactive thiol groups, which can be efficiently reduced back through the reaction catalyzed by $\operatorname{Trx}$ and peroxiredoxins, such as glutaredoxin $(\mathrm{GRX})^{4}$ (Figure 2).

Therefore, different from the pathological effects of heavy and severe oxidative stress, mild oxidative unbalance

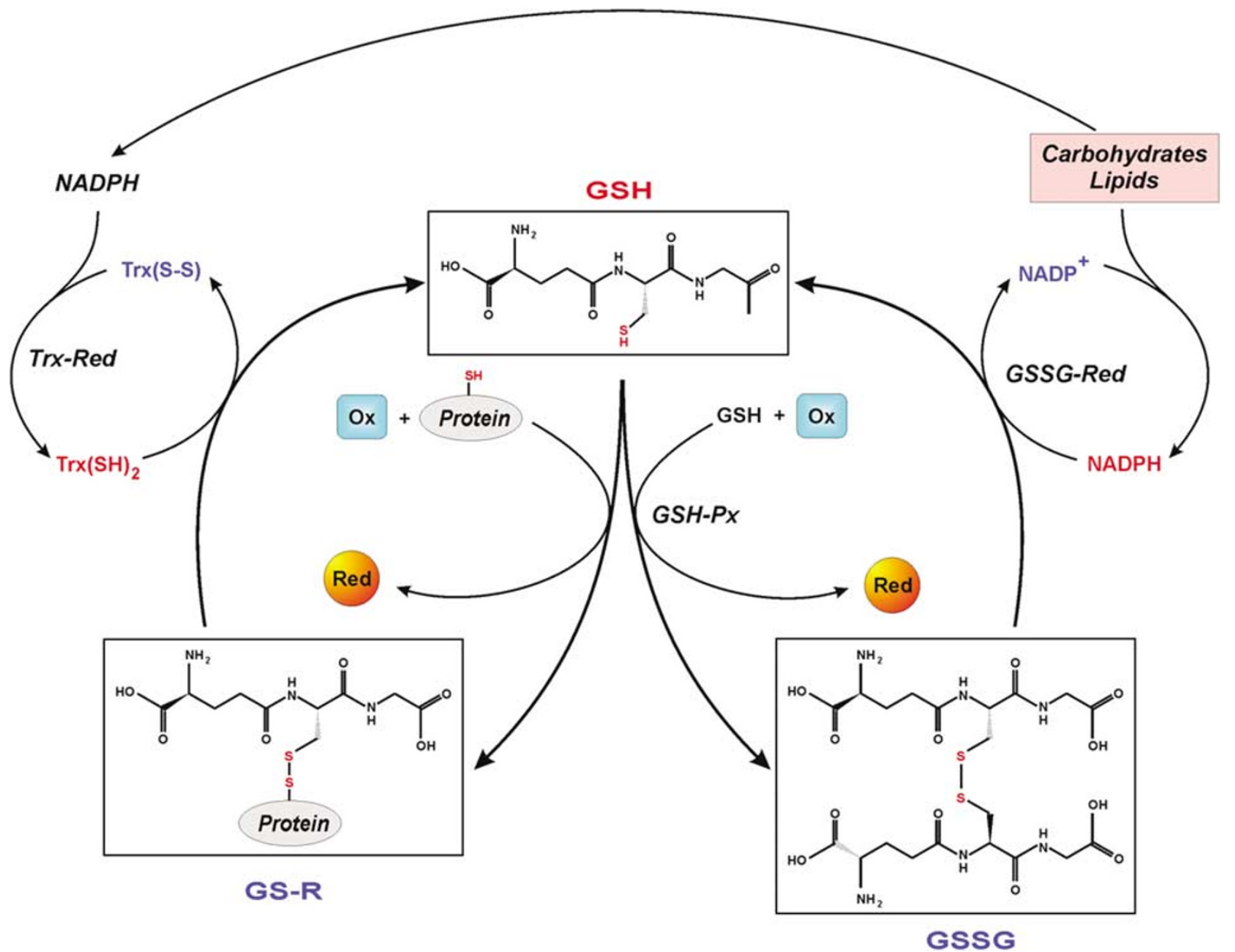

Figure 1 Glutathione redox cycle. In the schematic diagram are represented the reduced (GSH) and oxidized forms (GSSG and GS-R) of glutathione. The reduction back of GSSG and GS-R by the action of GSSG reductase (GSSG-Red) and thioredoxin (Trx) are also shown together with the contribution of NADPH as upstream supplier of reducing equivalents. (GSH-Px = Glutathione peroxidase) 
Table 1 Different modalities of oxidative stress

\begin{tabular}{|c|c|c|c|c|}
\hline [ROS] oxidative stress & Effect & Target & $\begin{array}{l}\text { Antioxidants implicated } \\
\text { in cell response }\end{array}$ & Response \\
\hline HEAVY (pathological) & $\begin{array}{l}\text { Irreversible oxidative } \\
\text { damage }\end{array}$ & $\begin{array}{l}\text { GENERAL } \\
\text { Cellular macromolecules } \\
\text { (Proteins, DNA, lipids) }\end{array}$ & $\begin{array}{l}\text { Enzymatic defense } \\
\text { (SOD, GPx, CAT) } \\
\text { GSH (cosubstrate and } \\
\text { scavenger) }\end{array}$ & $\begin{array}{l}\text { Nonprogrammed events: } \\
\text { 1. Rescue/survival } \\
\text { 2. NECROSIS }\end{array}$ \\
\hline MILD (physiological) & $\begin{array}{l}\text { Reversible oxidations } \\
\text { Regulation of gene } \\
\text { transcription }\end{array}$ & $\begin{array}{l}\text { SPECIFIC } \\
\text { Transcription (NF-kB, } \\
\text { AP-1, p53) } \\
\text { Regulatory proteins } \\
\text { (TRX, GST) }\end{array}$ & $\begin{array}{l}\text { GSH, TRX (mediators of } \\
\text { cell signaling) }\end{array}$ & $\begin{array}{l}\text { Programmed events: } \\
\text { 1. Proliferation } \\
\text { 2. APOPTOSIS }\end{array}$ \\
\hline
\end{tabular}

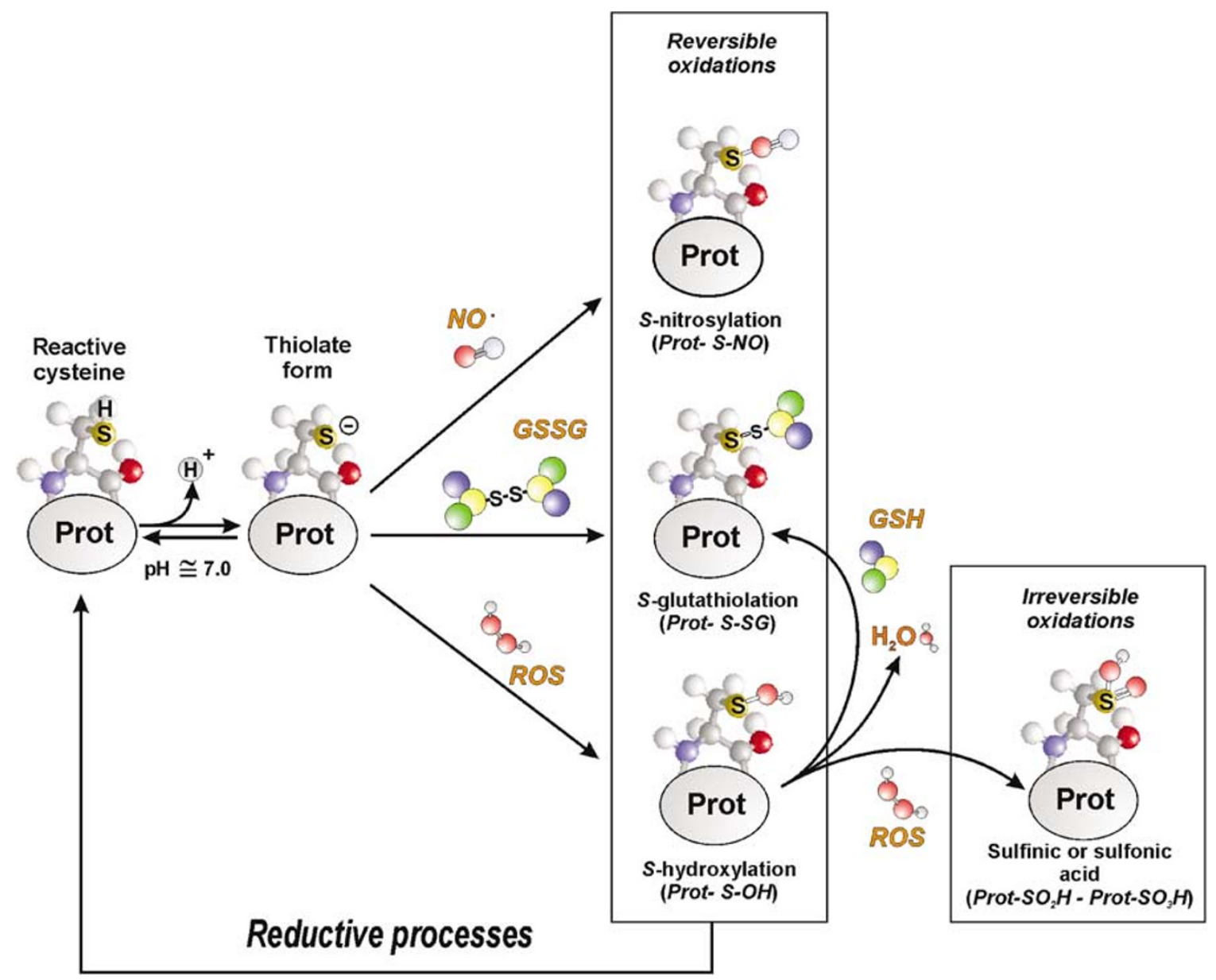

Thioredoxin/Glutaredoxin

Figure 2 Redox modifications of reactive cysteine. Under physiological pH, a reactive cysteine can exist as thiolate anion, which more easily undergoes reversible oxidation either by the reaction with ROS ( $S$-hydroxylation) or by the reaction of the NO radical (S-nitrosylation), or by the reaction with GSSG (S-glutathiolation). The last modification could also be the result of reaction between sulfenic acid derivative of cysteine (-SOH) and GSH. This reaction has been suggested to represent a protection acted by GSH to preserve enzyme functionality from further oxidations, which can determine irreversible modifications of cysteine. In fact, besides the only peroxiredoxins I-III, which have been demonstrated to undergo reversible sulfinylation, no reduction to thiol form can be catalyzed for sulfinic $\left(-\mathrm{SO}_{2} \mathrm{H}\right)$ and sulfonic $\left(-\mathrm{SO}_{3} \mathrm{H}\right)$ acid derivatives

represents a mode through which a redox signal can be transduced within the cell. The final effects of such redox alterations are programmed responses, worked out by the cellular machineries of proliferation, senescence or apoptosis.
In this perspective, the redox state of protein thiols represents a 'molecular switch' able to reversibly activate/deactivate protein function. This process resembles the well-established phosphorylative regulation (kinase/phosphatase) in which the 
addition/removal of a phosphate group reversibly changes protein activity.

Thiol-dependent redox-modulation of protein activities was firstly evidenced for transcription factors in bacteria. To survive in adverse environments, bacteria have evolved different and sophisticated mechanisms in order to rapidly modulate gene transcription to counteract several insults. Moreover, the absence of a physical barrier that isolates DNA makes the intracellular medium more efficient in buffering alterations of the extracellular environment. OxyR is a wellknown example of a redox responsive prokaryotic transcription factor. ${ }^{12}$ In particular, redox regulation of OxyR depends on the chemical status of specific cysteine residues and is responsive to $\mathrm{H}_{2} \mathrm{O}_{2}, \mathrm{SH}$-reactive compounds (i.e. diamide) and reactive nitrogen species (RNS). ${ }^{13,14}$ Pioneering studies by Storz and co-workers showed that, upon exposure to $\mathrm{H}_{2} \mathrm{O}_{2}$, the activation of OxyR involves the formation of a disulfide bond between Cys 199 and Cys 208, two residues that are separated by $17 \AA$ in the reduced - and inactive - form of the protein. The net outcome of this reaction is a change in DNA binding specificity, which occurs in a tetrameric conformation, and the recruitment of RNA polymerase. ${ }^{15}$ Recent findings from the Stamler laboratory demonstrate that, besides this 'on-off' modulation, a finer regulation for OxyR binding to DNA can occur. ${ }^{16}$ Only one thiol residue (Cys 199) is critical for OxyR activity and this residue can be differently oxidized depending on the extent of redox unbalance and the nature of the oxidizing molecules. The results obtained show that the redox stimuli differently affect its DNA binding, which is dependent on cooperative effects between OxyR subunits. Therefore, cysteine modification by $S$-nitrosylation (S-NO), S-glutathiolation (S-SG), intramolecular disulfide bond (S-S) formation, or oxidation to sulfenic acid (S-OH, S-hydroxylation) may be alternatively exploited to control gene expression. $^{16}$ OxyR is a model of direct and highly inducible regulation, being a quick response to cellular oxidative unbalance, which induces its binding to DNA in the oxidized form (Figure 3a).

While the response of prokaryotes to oxidative stress depends essentially on the 'duration' or 'moment' of exposure, eukaryotes have evolved a spatial regulation of transcription factors activity. In yeast, oxidative stress induces modifications that do not activate transcription activity directly, but interfere with the mechanisms of location of transcription factors to different cell compartments. A well-established example is represented by yeast activating protein 1 (Yap1), a functional homolog of the bacterial OxyR. ${ }^{17}$ Its deletion results in hypersensitivity not only to $\mathrm{H}_{2} \mathrm{O}_{2}$ but also to diamide, electrophiles and metals. Yap1 contains a noncanonical leucine-rich nuclear export signal (NES) embedded with the C-terminal cysteine-rich domain comprising of residues Cys 598, Cys 620 and Cys 629. Oxidative stress results in a disulfide bridge formation between the first two residues and in a conformational change that obstructs the NES, ${ }^{18}$ inhibiting export and leading to the accumulation of Yap1 in the nucleus, which results in its enhanced nuclear concentration and binding affinity to DNA ${ }^{19}$ (Figure $3 b$ ).

In multicellular organisms 'environment' is also, in a wider meaning, the relationship among cell populations through specialized signaling molecules such as hormones and

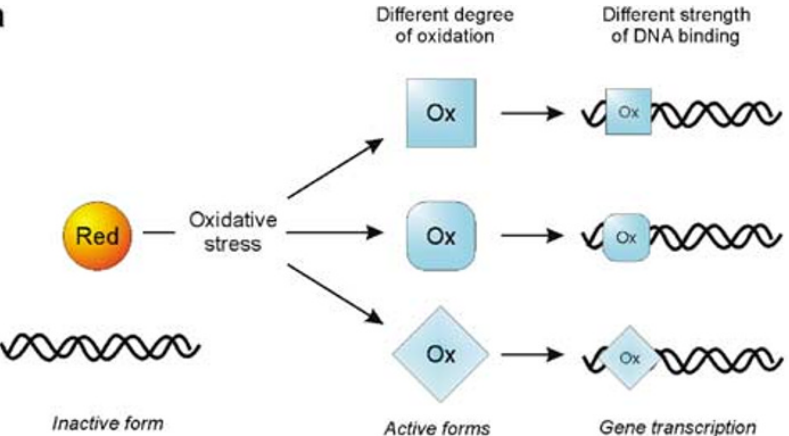

b

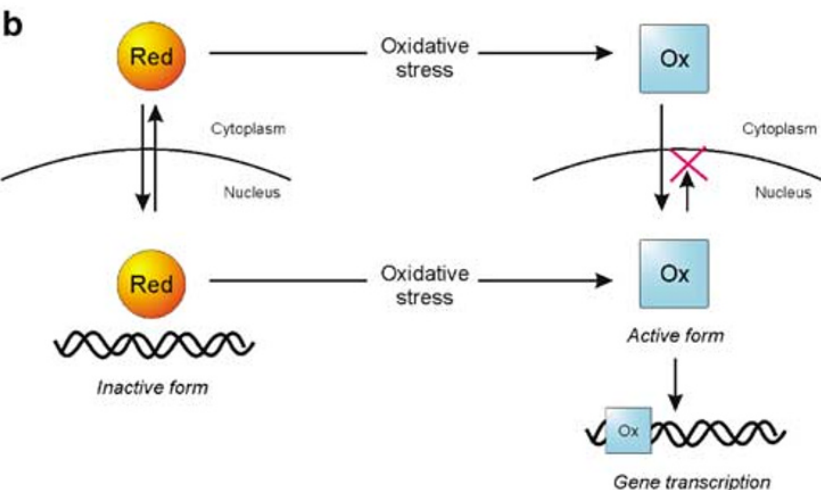

C

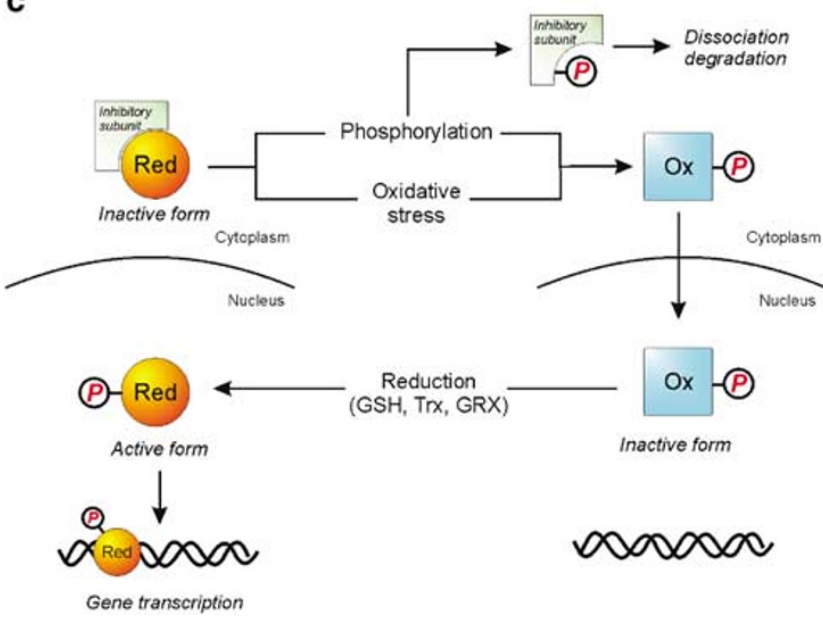

Figure 3 Redox regulation of gene transcription. (a) Prokaryotic redoxsensitive transcription factors bind to DNA only when oxidatively modified by the stimulus received. As demonstrated for $O x y R$, the strength of binding reflects the type of oxidative modification of reactive cysteines (S-OH, S-SG, S-NO). (b) Yeast transcription factors still bind to DNA upon oxidative modification; this reaction inhibits the exportation of the protein from the nucleus within the cytoplasm (e.g. Yap-1), thus increasing the affinity for DNA. (c) In higher eukaryotes, redox regulation does not represent the only way to modulate gene transcription. Often, under oxidative stimuli, it is accompanied by phosphorylation (directly to the transcription factor, such as AP-1; or to the inhibitory subunit, such as NF- $\kappa$ B). However, the activation of gene transcription needs a further reduction of transcription factors inside the nucleus to permit DNA binding. This double-step regulation (oxidation within the cytoplasm and reduction into the nucleus) allows the decrease of the risk of DNA mutation induced by oxidant conditions

growth factors. In this context, oxidative stress, from being an external challenge to cell survival takes up a further role in the regulation of many physiological processes. In particular, 
cell death represents a necessary event for the integrity and survival of cell populations, which, once induced by environmental challenge, has to occur in a gene-programmed manner (apoptosis). Changes of intracellular redox state becomes an additional signaling event that, besides buffering external oxidative challenge like in bacteria and yeast, can regulate proliferation, cell cycle and apoptosis. As a matter of fact, in multicellular eukaryotes, the activity of several transcription factors has been suggested to be modulated by redox changes of specific cysteines. Some of these redoxsensitive factors, such as $\mathrm{p} 53$, c-Jun/AP-1, nuclear factor- $\kappa \mathrm{B}$ $(\mathrm{NF}-\kappa \mathrm{B})$, and heat shock factor 1 (HSF1) are involved in the control of gene expression associated with cell cycle regulation and apoptosis. Many observations indicate that cells of different origin treated with oxidative stress-inducing agents, such as transition metals, oxyradicals, or thiol-reactive compounds, are efficiently activated for nuclear translocation. ${ }^{20}$ On the other hand, binding of transcription factors to DNA is permitted only upon reduction. In fact, in vitro experiments demonstrated that oxidative modification of specific cysteine residues could inhibit DNA binding. In particular, it has been reported that Cys 62 of the p50 subunit of $\mathrm{NF}-\kappa \mathrm{B}$ must be in reduced state in order to allow binding to promoter regions of its target genes. ${ }^{21}$ Cys 269 of c-Jun, closed to the interface of AP1 dimer, can undergo $S$ glutathiolation inhibiting AP-1 formation and DNA binding. ${ }^{22}$ Oxidation of Cys 182 of p53 to a mixed disulfide with GSH may prevent proper helix-helix interaction within the DNA binding domain and contribute to tetramer dissociation. ${ }^{23}$ These conclusions are supported by the evidence that the nucleus represents a very reducing compartment, in which GSH and Trx as well as redox sensitive factors such as Redox factor 1 (Ref-1), are present at high concentrations. In fact, Trx seems to translocate into the nucleus upon oxidative cell stimulation in order to modulate Ref-1, which, relying on a cysteine residue located at its $\mathrm{N}$-terminal end regulates the redox state of the above-mentioned transcription factors. ${ }^{24}$ HSF1 is also regulated by the intracellular redox state: a pro-oxidant environment favors its nuclear translocation, while DNA binding is increased by the reduction of critical cysteine residues. It has been demonstrated that $\mathrm{H}_{2} \mathrm{O}_{2}$ upregulates Trx before HSF1 activation indicating that Trx directly reduces HSF1 allowing its DNA binding during oxidative stress. $^{24}$

Such 'double step' regulation (oxidation within the cytosol and reduction inside the nucleus, Figure $3 c$ ) has two important consequences: (i) the nuclear compartment is sheltered from oxidative damage, which may have mutagenetic effects; (ii) gene transcription is activated only upon reversible oxidation of cysteine residues.

\section{Phosphorylative signaling}

The formation of a phospho-ester bond, by the action of protein kinases, is able to alter the function of proteins influencing protein conformations and in turn enzyme and cell function. ${ }^{25,26}$

The importance of signal transduction mediated by phosphorylative modification reflects in the high number of distinct protein kinase catalytic subunits (500-600). ${ }^{26}$ Phylogenetic analysis allows the protein kinase family to be divided into several subfamilies with finely tuned differences of sequence and structure, substrate selectivity and regulation properties. ${ }^{27}$ In eukaryotes, a well-known distinction is between the serine/threonine protein kinases (STPK), such as mitogen-activated protein (MAP) kinases, protein kinase $C(\mathrm{PKC})$, protein kinase $\mathrm{B}$ (Akt) and tumor growth factor- $\beta$ (TGF- $\beta$ ) receptor, and the tyrosine kinases (PTK), such as receptor tyrosine kinases (EGF-R, VEGF-R, IGF-R), phosphatidylinositol-3 kinase and Janus kinase. This rough classification gives no information about the mode of action through which phosphorylation occurs. Alternatively, protein kinases can be distinguished on the basis of the modulation of their catalytic activity, ${ }^{28}$ such as, for example auto-inhibition, in which the active site of the enzyme is occupied by a sequence located within the $\mathrm{N}$ - or $\mathrm{C}$-terminal extension of the catalytic core, which functions as a pseudosubstrate. Under this condition the activation of protein kinase relies on the capability of the substrate to displacing the autoinhibitory domain (or a distinct subunit of the enzyme) from the active site. This type of regulation characterizes the binding of $\mathrm{Ca}^{2+} /$ calmodulin to the autoinhibitory domains of both myosin light chain kinase and calmodulin-dependent kinase II, the binding of $\mathrm{Ca}^{2+}$ to the auto-inhibitory domain of PKC and phosphorylation of the C-terminal tail of the SRC tyrosine kinase. A second class of kinase regulation is represented by the phosphorylation within the kinase catalytic core, the target site being a conserved threonine or tyrosine residue localized within a defined active site segment, termed the activation loop. In this case, the principal role of phosphorylation is to correctly remodeling the active site of the enzyme as to optimize substrate binding and chemical transfer of the phosphoryl group. MAP kinases and cyclin-dependent kinases (CDK) are peculiar members of this class of enzymes, which undergo phosphorylation and activation by heterologous kinases.

In eukaryotes, phosphorylation represents a 'cascade' mode of signaling, as by activating a few number of protein kinases, a highly inducible process is switched on. In fact, the reactions catalyzed by these enzymes often represent the start for the subsequent activation of other members of the same family. Therefore, the chain reaction, which derives from the transformation of the proform into the catalytically active specie of single kinases, can propagate within the cell as a wave of activation. In this way, even a little stimulus can induce activation of a kinase network and this is fundamental for an effective and rapid cell response.

A highly organized system such as that of protein kinases needs a similarly complex counterpart to be deactivated. Several protein phosphatases governs these processes and, being intimately related to the kinase network, are often activated by the same phosphorylative events or able to recognize and dephosphorylate only the phosphoryl-conjugate isoform of protein kinases. The capability of these processes to be modulated by single (or more) addition of phosphoryl group(s) causes a rapid and easy modulation of response. This signaling has been often reported to be activated in response to stimuli involved in cell cycle progression and/or apoptosis. 


\section{Comparison between redox- and phospho-mediated signaling}

By dissecting the mechanisms through which redox- and phosphorylation-mediated transduction evolved, we can see similarities and distinctions. One aspect to be considered is specificity. In fact, in the redox-mediated signal transduction, the oxidative modification of a cysteine is conceivable only if the chemical environment in which the residue is located determines a decrease in the $\mathrm{p} K_{\mathrm{a}}$ that favor deprotonation of sulfhydryl (-SH) into the thiolate $\left(-\mathrm{S}^{-}\right)$form. ${ }^{10}$ This requirement implies that only one or few cysteines become reactive. Oxidative modifications of specific reactive cysteines seem to be highly selective as they depend on the tri-dimensional structure of each protein. At the same time, phosphorylation of serine/threonine or tyrosine residues does not occur if specific protein/protein recognition does not take place. However, even if the specificity of phosphorylation reaction has been extensively addressed, this feature appears to be not so stringent. In fact, a great deal of evidence demonstrated that besides the specific substrates, protein kinases could also phosphorylate other proteins that not necessarily show surface complementarities if not preceded by conformational rearrangement. This is the case of the c-Jun N-terminal kinase (JNK) for which the capability to phosphorylate p53 and B-cell lymphoma-2 (Bcl-2) besides c-Jun has been evidenced. ${ }^{29,30}$ This feature seems to be related to the proapoptotic function of JNK, so that its activity at the same time can inhibit MDM-2-mediated p53 degradation and activate $\mathrm{Bcl}-2$ proteolytic process.
A similarity between phospho- and redox-mediated signaling is the reversibility of the process. For example, $S$ hydroxylation or S-glutathiolation are transient modifications that could be reversed by the action of GRX or Trx. ${ }^{4}$ Analogously, once activated by phosphorylation, protein kinases can return to the inactive form through the action of specific protein phosphatases (Table 2).

On the other hand, the most important difference between the two signaling pathways is the modality of cell response upon ensuing the stimulus. The phosphorylative event is an active process because the transfer of a phosphoryl group requires the hydrolysis of ATP. The downstream effects of phosphorylative events are, often, represented by sequential steps of phospho-activation. Such cascade of reactions draws a coordinated network that might be controlled at multiple levels. This energy-dependent mode of protein activation underlines the 'strength' of the signal, so that the phosphorylate form of the target protein is maintained until phosphatases action occur. On the contrary, redox signaling could be defined as a buffer response to the oxidation potential of the cell. This may also explains why redox response is energyindependent and proportional to the level of the oxidative burst: more concentrated are the intracellular pro-oxidant species (disulfides and/or ROS) more effective will be the oxidative modification of sensitive proteins. It can be stated that the modulation of redox-mediated transduction pathway is a unidirectional straight-line process, not a cascade, as the final modification of the target sensitive protein terminates the process (ON/OFF switch) (Table 3).

Table 2 Similarities between redox and phosphorylative signaling

\begin{tabular}{|c|c|}
\hline $\begin{array}{l}\text { Redox } \\
+++\end{array}$ & $\begin{array}{l}\text { Phospho } \\
++\end{array}$ \\
\hline $\begin{array}{l}\text { Single aminoacid (cys) } \\
\text { Sensitivity to oxidation (chemical environment) }\end{array}$ & $\begin{array}{l}\text { polar -OH aminoacids (Ser-Thr) } \\
\text { Protein/protein recognition (external surface) }\end{array}$ \\
\hline $\begin{array}{l}\text { Redox } \\
++\end{array}$ & $\begin{array}{l}\text { Phospho } \\
+++\end{array}$ \\
\hline $\begin{array}{l}\text { Enzyme dependent (trx/tnx-Red-Grx/Grx-Red) } \\
\text { Energy-dependent (NADPH-reducing power) }\end{array}$ & $\begin{array}{l}\text { Enzyme dependent (protein phosphatases-PP) } \\
\text { Energy-dependent (ATP-PP phosphoactivation) }\end{array}$ \\
\hline
\end{tabular}

Table 3 Differences between redox and phosphorylative signaling

\begin{tabular}{|c|c|}
\hline Redox & Phospho \\
\hline $\begin{array}{l}\text { 'Buffer' response } \\
\text { Passive } \\
\text { Energy independent } \\
\text { Proportional to the oxidation degree }\end{array}$ & $\begin{array}{l}\text { 'Explosive' response } \\
\text { Active } \\
\text { Energy dependent } \\
\text { Highly inducible }\end{array}$ \\
\hline \multicolumn{2}{|c|}{ Downstream effects } \\
\hline $\begin{array}{l}\text { Direct modulation } \\
\quad \text { Target protein is the final effector of signaling } \\
\downarrow \\
\text { Unidiretionai one-step modulation (ON/OFF) }\end{array}$ & $\begin{array}{l}\text { Cascade reaction } \\
\text { Sequential steps of phosphoactivation } \\
\text { Coordinated network (control at multiple steps) }\end{array}$ \\
\hline
\end{tabular}




\section{Cross talk between redox and phosphorylative processes}

Cross talk and/or sequential partnership between two different signaling mechanisms have been recently found to occur in some cases of cell regulation. Trx/ASK1 was the first system identified by Saitoh and co-workers where a redox switch resulted in a phosphorylative process. ${ }^{31}$ Apoptosis signalregulating kinase (ASK1) belongs to the stress-activated protein kinases (SAPK) family that regulates the activation of different MAP kinase kinases (MKK). ASK1 has been suggested to activate p38- ${ }^{\mathrm{MAPK}}$ and JNK-upstream kinases, MKK $3 / 6$ and MKK 4/7, respectively, ${ }^{32}$ and can be activated by chemical and physical stress, as well as through stimulation of death receptors. Studies using the yeast two-hybrid system led to identify Trx as an ASK1 interacting regulatory protein. ${ }^{31}$ The association between Trx and ASK1 gives rise to a dimer Trx/ASK1, which inactivate the kinase activity of ASK1; moreover, this interaction was found only in nonstressed cells and seemed to be modulated by the intracellular redox state. In particular, a more oxidizing environment has been suggested to cause disulfide bridge formation on the Trx moiety thus destabilizing the dimer. As a result, ASK1 can escape from Trx inhibition and undergo multimerization, which corresponds to the active form of the enzyme. ${ }^{33}$ As already mentioned, Trx is a small protein implicated in a variety of cellular functions such as correct folding of proteins inside the endoplasmic reticulum, activation of $T$ lymphocytes after the recognition of antigen-presenting cells, and reduction of ribonucleotide reductase. Trx possess two vicinal cysteines forming intra- or intermolecular disulfide bridge. The first characterization of this tightly regulated system was carried out in the presence of high concentrations of $\mathrm{H}_{2} \mathrm{O}_{2}$, but later on it has been demonstrated that an increase in intracellular disulfide potential, in terms of mixed disulfides between GSH and protein thiols, could also induce the dissociation of Trx from ASK1 and downstream activate the phosphorylative cascade that ultimately induces transcription of several genes involved in the regulation of cell cycle and apoptosis ${ }^{34}$ (Figure 4).

The second system, for which a strict association between redox unbalance and phosphorylative events has been found, is represented by the glutathione transferase $\pi-1$ (GST)/JNK complex. JNK activity is physiologically maintained at low levels within the cell, even in the presence of high concentrations of growth factors and it seems to be inhibited in unstressed cells. Adler and co-workers purified the inhibitory component of JNK and identified it as GST. ${ }^{35}$ They demonstrated that under resting conditions, JNK phosphorylation is inhibited by its association with GST, while under $\mathrm{H}_{2} \mathrm{O}_{2}$ or UV

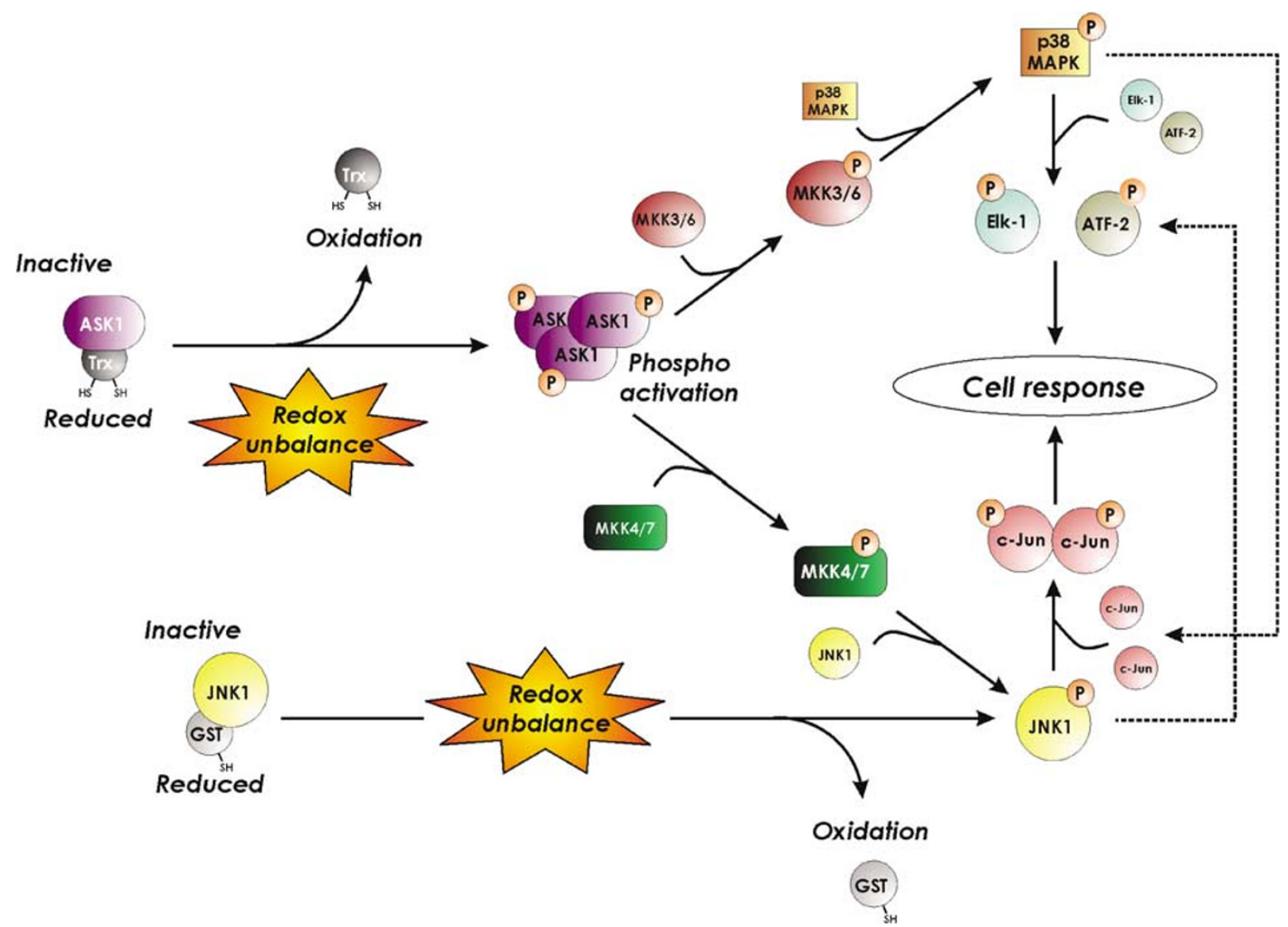

Figure 4 Redox regulation of MAP kinases. The scheme represents the intimate relationship between phospho- and redox-mediated signals. The detachment of the redox-sensitive inhibitory subunits, Trx and GST, from ASK1 and JNK, respectively, allows transforming an oxidative stimulus in a phosphorylative cascade 
treatment, the GST/JNK complex dissociates leading to formation of GST dimer and/or multimers and phosphoactivation of $\mathrm{JNK}^{36}$ The dissociation process seems to be highly affected by oxidative conditions, even though rapid and of low intensity, is able to induce a reversible dissociation between the two subunits ${ }^{37}$ (Figure 4).

The identification of Trx and GST proteins as modulators of ASK1 and JNK activities provides strong evidence for the mechanism through which a redox-mediated signaling event could be strictly connected to downstream processes mediated by stress kinases activation. In particular, increased disulfide potential leads to activation of protein kinases and, in some cases, to induction of apoptosis.

Protein phosphatases appear to be regulated by redox reaction as well. Protein tyrosine phosphatases (PTPases) activity is highly regulated by oxidation/reduction reactions involving the cysteine residue required for catalysis. ${ }^{38}$ PTPases share a conserved 230-amino-acid domain containing the cysteine residue that catalyzes the hydrolysis of phosphate from phospho-tyrosine residues by the formation of a cysteinyl-phosphate intermediate. PTPases are specifically involved in the regulation of reversible tyrosine phosphorylation in the insulin action pathway. Recently, it has been established that insulin stimulation generates a burst of intracellular $\mathrm{H}_{2} \mathrm{O}_{2}$ in insulin-sensitive cells, which is associated with reversible oxidative inhibition of overall cellular PTPase activity, especially the PTP-1B family member. ${ }^{38}$ It can be concluded that oxidative alterations are able to function as positive regulators of phosphorylation-mediated apoptosis by activating protein kinases, such as JNK and ASK1, and, at the same time, by inducing a reversible loss of function of PTPases.

\section{Evolutionary hypotheses}

Different types of post-translational modifications, such as glycosylation, methylation and protein cleavage, have been selected in order to allow an extracellular stimulus to be transduced through cellular districts. They depend on the formation of protein/protein complexes, in which one partner represents the modifying enzymatic subunit (glycosylase, methylase, protease) while the other partner is the receiving protein that, once modified, can function within the cell. Among these modifications, oxidation and phosphorylation differ in significant aspects. The former process needs no enzymatic counterpart; therefore, proteins that are oxidized on critical cysteines residues are modulated in their function directly by the stimulus applied (i.e. oxidative stress). Conversely, during phosphorylation-mediated signal transduction, the receiving protein is often represented by another protein kinase that, once modified, behaves as the modifying enzyme of proteins further downstream, which could be other protein kinases. In this case, the presence of several steps of modification (cascade) makes the signal amplified and monitored at multiple levels.

We can argue that cysteine oxidation, selected by prokaryotes as defense against oxidizing environments, and then maintained by eukaryotes owing to its high specificity, may represent an ancestral way of signaling. On the other hand, phosphorylation represents the most used eukaryotic modality of signaling because of its redundancy and amplification degree. The discovery of the MAP kinases-mediated transduction pathways has shown a synergism between redox and phospho-signaling. In particular, the upstream control of the system is managed by the specificity of reactive cysteines oxidation, and the downstream propagation of the signal is handled by phosphorylative chain reactions. This highly regulated molecular mechanism suggests that other systems could be modulated in this way. Interestingly, it has been recently suggested that, as evidenced in bacteria for OxyR regulation, different types of oxidative stress - alteration of intracellular GSH/GSSG ratio or increase in ROS production - could induce the activation of different MAP kinases. In particular, while Trx/ASK1/p38 ${ }^{\mathrm{MAPK}}$ system seems to be sensitive to increase of cellular disulfides, ${ }^{34,39,40}$ GST/JNK system seems to be modulated by increases of ROS concentration. ${ }^{40}$ The selective activation of MAP kinases and the downstream induction of apoptosis, in response to oxidative modifications of different types, could also be a hystotype-dependent feature, which needs further investigation, as it could open a new avenue of research in the chemotherapeutic strategies against growth of cancer cells and neurodegenration.

\section{References}

1. McCord JM and Fridovich I (1969) Superoxide dismutase. An enzymic function for erythrocuprein (hemocuprein). J. Biol. Chem. 244: 6049-6055

2. Finkel $\mathrm{T}$ and Holbrook NJ (2000) Oxidants, oxidative stress and the biology of ageing. Nature 6809: 239-247

3. Schafer FQ and Buettner GR (2001) Redox environment of the cell as viewed through the redox state of the glutathione disulfide/glutathione couple. Free Radical Biol. Med. 30: 1191-1212

4. Filomeni $G$, Rotilio $G$ and Ciriolo MR (2002) Cell signalling and the glutathione redox system. Biochem. Pharmacol. 64: 1057-1064

5. Rotilio G, Mavelli I, Rossi L and Ciriolo MR (1985) Biochemical mechanism of oxidative damage by redox-cycling drugs. Environ. Health Perspect. 64: 259264

6. Akhand AA, Du J, Liu W, Hossain K, Miyata T, Nagase F, Kato M, Suzuki H and Nakashima I (2002) Redox-linked cell surface-oriented signaling for T-cell death. Antioxidants Redox Signal. 4: 445-454

7. Nakamura H, Nakamura K and Yodoi J (1997) Redox regulation of cellular activation. Annu. Rev. Immunol. 15: 351-369

8. Kamata H, Honda S, Maeda S, Chang L, Hirata H and Karin M (2005) Reactive oxygen species promote TNFalpha-induced death and sustained JNK activation by inhibiting MAP kinase phosphatases. Cell 120: 649-661

9. Takeda K, Kato M, Wu J, Iwashita T, Suzuki H, Takahashi M and Nakashima I (2001) Osmotic stress-mediated activation of RET kinases involves intracellular disulfide-bonded dimer formation. Antioxidants Redox Signal. 3: 473-482

10. Finkel $T$ (2000) Redox-dependent signal transduction. FEBS Lett. 476: $52-54$

11. Barford $D(2004)$ The role of cysteine residues as redox-sensitive regulatory switches. Curr. Opin. Struct. Biol. 14: 679-686

12. Helmann JD (2002) OxyR: a molecular code for redox sensing? Sci. STKE 5 : PE46

13. Paget MS and Buttner MJ (2003) Thiol-based regulatory switches. Annu. Rev. Genet. 37: 91-121

14. Georgiou $G$ (2002) How to flip the (redox) switch. Cell 111: $607-610$

15. Zheng M, Aslund F and Storz G (1998) Activation of the OxyR transcription factor by reversible disulfide bond formation. Science 279: 1718-1721

16. Kim SO, Merchant K, Nudelman R, Beyer Jr WF, Keng T, DeAngelo J, Hausladen A and Stamler JS (2002) OxyR: a molecular code for redox-related signaling. Cell 109: 383-396 
17. Toone WM, Morgan BA and Jones N (2001) Redox control of AP-1-like factors in yeast and beyond. Oncogene 20: 2336-2346

18. Delaunay A, Pflieger D, Barrault MB, Vinh J and Toledano MB (2001) A thiol peroxidase is an $\mathrm{H}_{2} \mathrm{O}_{2}$ receptor and redox-transducer in gene activation. Cell 111: $471-481$

19. Kuge S, Arita M, Murayama A, Maeta K, Izawa S, Inoue $Y$ and Nomoto A (2001) Regulation of the yeast Yap1p nuclear export signal is mediated by redox signal-induced reversible disulfide bond formation. Mol. Cell. Biol. 21: 6139-6150

20. Marshall HE, Merchant $\mathrm{K}$ and Stamler JS (2000) Nitrosation and oxidation in the regulation of gene expression. FASEB J. 14: 1889-1900

21. Pineda-Molina E, Klatt P, Vazquez J, Marina A, Garcia de Lacoba M, PerezSala D and Lamas S (2001) Glutathionylation of the p50 subunit of NF-kappaB: a mechanism for redox-induced inhibition of DNA binding. Biochemistry 40: 14134-14142

22. Klatt $P$, Molina EP, De Lacoba MG, Padilla CA, Martinez-Galesteo E, Barcena JA and Lamas S (1999) Redox regulation of C-Jun DNA binding by reversible S-glutathiolation. FASEB J. 13: 1481-1490

23. Sun XZ, Vinci C, Makmura L, Han S, Tran D, Nguyen J, Hamann M, Grazziani S, Sheppard S, Gutova M, Zhou F, Thomas J and Momand J (2003) Formation of disulfide bond in p53 correlates with inhibition of DNA binding and tetramerization. Antioxidants Redox Signal. 5: 655-665

24. Jacquier-Sarlin MR and Polla $B$ (1996) Dual regulation of heat shock transcription factor (HSF) activation and DNA-binding activity by $\mathrm{H}_{2} \mathrm{O}_{2}$ : role of thioredoxin. Biochem. J. 318: 187-193

25. Pawson T (1994) Introduction: protein kinases. FASEB J. 8: 1112-1113

26. Hunter $T$ (1987) A thousand and one protein kinases. Cell 50: 823-829

27. Hanks SK, Quinn AM and Hunter T (1988) The protein kinase family: conserved features and deduced phylogeny of the catalytic domains. Science $241: 42-52$

28. Johnson LN, Noble ME and Owen DJ (1996) Active and inactive protein kinases: structural basis for regulation. Cell 85: 149-158

29. Buschmann T, Potapova O, Bar-Shira A, Ivanov VN, Fuchs SY, Henderson S, Fried VA, Minamoto T, Alarcon-Vargas D, Pincus MR, Gaarde WA, Holbrook NJ, Shiloh Y and Ronai Z (2001) Jun NH2-terminal kinase phosphorylation of p53 on Thr-81 is important for p53 stabilization and transcriptional activities in response to stress. Mol. Cell. Biol. 21: 2743-2754
30. Schroeter H, Boyd CS, Ahmed R, Spencer JP, Duncan RF, Rice-Evans C and Cadenas E (2003) c-Jun N-terminal kinase (JNK)-mediated modulation of brain mitochondria function: new target proteins for JNK signalling in mitochondriondependent apoptosis. Biochem. J. 372: 359-369

31. Saitoh M, Nishitoh H, Fujii M, Takeda K, Tobiume K, Sawada Y, Kawabata M, Miyazono K and Ichijo H (1998) Mammalian thioredoxin is a direct inhibitor of apoptosis signal-regulating kinase (ASK) 1. EMBO J. 17: 2596-2606

32. Ichijo $\mathrm{H}$, Nishida E, Irie $\mathrm{K}$, ten Dijke $\mathrm{P}$, Saitoh M, Moriguchi T, Takagi M, Matsumoto K, Miyazono K and Gotoh Y (1997) Induction of apoptosis by ASK1, a mammalian MAPKKK that activates SAPK/JNK and p38 signaling pathways. Science 5296: 90-94

33. Liu $Y$ and Min W (2002) Thioredoxin promotes ASK1 ubiquitination and degradation to inhibit ASK1-mediated apoptosis in a redox activity-independent manner. Circ. Res. 90: 1259-1266

34. Filomeni G, Rotilio G and Ciriolo MR (2003) Glutathione disulfide induces apoptosis in U937 cells by a redox-mediated p38 MAP kinase pathway. FASEB J. 17: $64-66$

35. Adler V, Yin Z, Fuchs SY, Benezra M, Rosario L, Tew KD, Pincus MR, Sardana M, Henderson CJ, Wolf CR, Davis RJ and Ronai Z (1999) Regulation of JNK signaling by GSTp. EMBO J. 18: 1321-1334

36. Adler V, Yin Z, Tew KD and Ronai Z (1999) Role of redox potential and reactive oxygen species in stress signaling. Oncogene 18: 6104-6111

37. Filomeni G, Aquilano K, Rotilio G and Ciriolo MR (2003) Reactive oxygen species-dependent c-Jun $\mathrm{NH} 2$-terminal kinase/c-Jun signaling cascade mediates neuroblastoma cell death induced by diallyl disulfide. Cancer Res. 63: $5940-5949$

38. Barrett WC, DeGnore JP, Konig S, Fales HM, Keng YF, Zhang ZY, Yim MB and Chock PB (1999) Regulation of PTP1B via glutathionylation of the active site cysteine 215. Biochemistry 38: 6699-6705

39. Turella P, Cerella C, Filomeni G, Bullo A, De Maria F, Ghibelli L, Ciriolo MR, Cianfriglia M, Mattei M, Federici G, Ricci G and Caccuri AM (2005) Proapoptotic activity of new glutathione S-transferase inhibitors. Cancer Res. 65: 3751-3761

40. Filomeni G, Aquilano K, Civitareale P, Rotilio G and Ciriolo MR (2005) Activation of c-Jun-N-terminal kinase is required for apoptosis triggered by glutathione disulfide in neuroblastoma cells. Free Radical Biol Med 39: $345-354$ 